\title{
Capsule Commentary on Luu et al., Provider-to-Provider Communication During Transitions of Care From Outpatient to Acute Care: A Systematic Review
}

\author{
Thomas D. Harter, PhD \\ Gundersen Health System, La Crosse, WI, USA. \\ J Gen Intern Med 31(4):414 \\ DOI: $10.1007 / \mathrm{s} 11606-016-3585-6$ \\ (c) Society of General Internal Medicine 2016
}

$\mathrm{T}$ his literature review by Luu et al. ${ }^{1}$ examines the results of 20 studies about provider-to-provider communication for the transfer of persons from outpatient to inpatient care settings. The authors narrowed their results from 4009 initial citations by including only original articles written in English, involving human subjects, and that were clearly about transitions of care or direct provider-to-provider communication. The result is a small cohort of studies from the US, the UK, and Australia, through which the authors explore the frequency and type of communication methods used during transitions of care from outpatient to inpatient settings. They found that most of these studies themselves were small and presented statistically insignificant data about communication outcomes, and thus conclude that the existing evidence regarding outpatient-to-inpatient provider-to-provider communication is insufficient.

The authors attempt to address and evaluate what is known about provider-to-provider communication during outpatient-to-inpatient care transitions and the potential effects of direct provider-to-provider communication on certain outcomes, including mortality, readmissions, length of stay, patient and provider satisfaction, and utilization. Unfortunately, the study's size and approach limit its overall value, except to note in the conclusion that more robust studies about outpatient-to-inpatient provider-to-provider communication are needed. It is also unclear how the noted outcome measures were identified, or that they are capable

of defining ideal communication methods for optimizing patient care and minimizing potential medical errors during outpatient-to-inpatient transitions. Many variables that occur after an outpatient-to-inpatient transition, and that are unrelated to the initial communication, can affect all the identified outcome measures.

Other unexamined pieces would have been helpful to address, such as to what degree provider communication plays a role in determining whether transitions from outpatient to inpatient care settings align with patient wishes, and whether such communications affect treatment decision-making at the moment of inpatient admission. At least mentioning the potential influences of macro- and micro-systematic differences in the provision of health care between the US, the UK, and Australia would also be helpful in contextualizing the importance and impact of communication with outpatient-toinpatient care transitions. Future work should also study how the reasons for outpatient-to-inpatient transfers are communicated between providers.

Corresponding Author: Thomas D. Harter, PhD; Gundersen Health System, La Crosse, WI, USA (e-mail: tdharter@gundersenhealth.org).

Compliance with Ethical Standards:

Conflict of Interest: The author declares that he does not have a conflict of interest.

\section{REFERENCE}

1. Luu NP, Pitts S, Petty B, Sawyer MD, Dennison-Himmelfarb C, Boonyasai RT, Maruthur NM. Provider-to-provider communication during transitions of care from outpatient to acute care: a systematic review. J Gen Intern Med. 2016. doi:10.1007/s11606-015-3547-4.

This comment refers to the article available at: $h t t p: / / d x$. doi.org/10.1007/ s11606-015-3547-4.

Published online January 19, 2016 